the products of either bacteria or viruses ${ }^{2}$, raising the obvious possibility that the IDDM-associated superantigen is of infectious origin. In this regard there is evidence for upregulation of expression of endogenous retroviruses in diseased pancreatic $\beta$-cells in certain mouse models ${ }^{12}$, and infection with some viruses (such as congenital rubella) increases the risk of IDDM in genetically susceptible individuals $^{13}$. As Conrad et al. point out, the putative infectious agent encoding the superantigen could either reside in pancreatic $\beta$-cells or alternatively be transported to the islets by infected lymphocytes. Whatever the explanation, the race will soon be on to identify this islet superantigen and to determine more precisely its role in the aetiology of IDDM.

H. Robson MacDonald and Hans AchaOrbea are at the Ludwig Institute for Cancer Research, Lausanne Branch, University of Lausanne, 1066 Epalinges, Switzerland.

\title{
Snap-together vesicles
}

\section{Helmut Ringsdorf and Joachim Simon}

IN cell membranes, mother nature demonstrates the perfect interplay of molecular recognition and molecular selforganization to assemble systems whose function is based on their organization. Science has lagged far behind. Until recently, attempts to use the principles of self-organization, regulation, replication, communication and cooperativity to build up structures have remained at the level of systems such as monolayers, vesicles or micellar aggregates. Interest is growing in molecular engineering of the next level of supramolecular systems where already complex selforganized aggregates are combined to yield larger functional assemblies. The aim is to mimic the fascinating pathways of nature where molecules, macromolecules and molecular devices can be seen as merely the building blocks for cells, cell complexes, organs and organisms.

Chiruvolu et al. ${ }^{1}$ have now combined two well-known and well characterized model systems, namely vesicles and biotin-streptavidin as recognition-units. They have looked into a neglected application for these systems: the formation of cross-linked vesicular aggregates by site-specific recognition.

First, they prepared small unilamellar vesicles from mixtures of naturally occurring phospholipids and biotinfunctionalized lipids. Biotin (vitamin $\mathrm{H}$ ) binds to a tetravalent protein (Streptavidin) from the bacterium Streptomyces avidinii with a binding constant of $k_{\mathrm{a}}=$ $10^{15} \mathrm{M}^{-1}$. The popularity of this system originates from its widespread usage in affinity chromatography ${ }^{2}$. Upon addition of the tetravalent protein to the vesicular dispersion, the vesicles aggregate and precipitate, as shown schematically in the figure.

This recognition experiment is thus far merely a variation on a theme that has been extensively investigated in liposomes ${ }^{3}$ and monolayers ${ }^{4,5}$. Only the lipid structure and vesicle composition were slightly changed. But it became very interesting upon closer investigation. Cryo-microscopical examination of the aggregates revealed tethered liposomes

encapsulation composites.

With the new, site-specific binding between vesicles, the build-up of secondary supramolecular structures looks to be within reach, especially as the composition and thereby the distribution of binding sites in vesicular systems is easily controllable. And the aggregation is reversible: on competitive replacement of the lipid-bound biotin, the liposome aggregates could be redispersed without losing the integrity of the vesicular compartments. One of us (H.R.) found similar results several years ago using coloured vesicles prepared from polydiacetylene lipids with sugar headgroups. The coloration allowed us to follow the reversible aggregation of the intact vesicles by specific binding of lectins such as Concanavalin $\mathrm{A}^{8}$. Chiruvolu has calculated the binding forces between the tethered liposomes, and found that one specific binding site between two liposomes is energetically comparable to the strongest binding interactions attainable with nonspecific colloidal forces ${ }^{9}$ - but without the disadvantages that nonspecific aggregations bring about.

Specific, reversible crosslinking of vesicular assemblies looks promising as part of experiments designed to examine specific binding events and binding forces ${ }^{10}$, as the size of the vesicles, their degree of functionalization or the inclusion of probes can be easily controlled ${ }^{11}$. Even more exciting would be the possibility of constructing soft biomaterials with tunable properties. Crosspolymerization of the lipids, and the use of ligandfunctionalized hydrophilic polymers as cross-linking matrices, can strengthen and sta-

linked to each other by biotinstreptavidin-biotin bridges, but which have retained their spherical shapes and seemed to have mostly unperturbed membrance surfaces.

In contrast, liposomes that have been aggregated under osmotic stress or by addition of calcium ions ${ }^{6,7}$ have to undergo dramatic deformations to maximize the attractive forces between single vesicular compartments. Such deformations make the vesicle membranes fragile and leaky, and sometimes lead to fusion of membranes, reducing the prospect of using assemblies of vesicles, for example as

1. Chiruvolu. S. etal. Science 264, 1753-1755 (1994)

2. Bayer, E. A.\& Wilchek. M. (eds) Avidin-Biotin Technology, Methods in Enzymology, Vol. 184 (Academic, San Diego, 1990)

3. Müller, W. etal. Science 262, 1706-1708 (1993)

4. Ahiers, M., Müller, W., Reichert, A., Ringsdorf, H.\& Venzmer, J. Angew. Chem. 102, 1310-1327 (1990)

5. McConnell, H. M., Watts, T. H., Weiss, R. M. \& Brian, A. A. Biochim. biophys. Acta 864, 95-106 (1986).

6. Heim. C. A., Israeiachvili, J. N. \& McGuiggan, P. M. Biochemistry 31, 1794-1805 (1992). bilize the assembly but allow us to retain control of the bulk properties by manipulating osmotic forces - swelling or shrinking will influence the elastic properties of the material. Innumerable possibilities exist if we can combine liposomes or polymerized liposomes with polymeric materials. It will be exciting to follow whether these concepts will finally lead to artificial tissue-like composites.

Helmut Ringsdorf and Joachim Simon are in the Institut für Organische Chemie, Johannes Gutenberg-Universität, 55099 Mainz, Germany.

7. Hui, S. W., Nio, S., Stewart, T. P., Boni. L. T. \& Huang, S. K. Biochem. biophys. Acta 941, 130-140 (1988).

8. Bader, H., Dorn, K., Hupfer, B. \& Ringsdorf, H. Adv. Polymer Sci. 64, 1-61 (1985).

9. Israelachvili, J. N. Intermolecular and Surface Forces (Academic, London, 1992).

10. Florin, E., Moy, V. \& Gaub, H. Science 264, 415-417 (1994).

11. Gregoriadis, G. Liposome Technology, Vol. I-III (CRC Boca Raton, 1984) 